\title{
Reduction of Feedback Based-on User Traffic Rate in OFDM Relay System
}

\author{
Qian-Bin Chen, Guang-Jian Jiang, Lun Tang \\ Chongqing Key Lab of Mobile Communication Technology, Chongqing University of Posts and \\ Telecommunications, Chongqing China
}

\begin{abstract}
In order to reduce feedback while maintaining user performance in multiuser orthogonal frequency division multiplexing (OFDM) relay system, feedback mechanism based on user traffic rate is proposed. From the aspects of feedback outage probability, user weighted channel gain related to user traffic rate is compared with the system threshold. The higher user traffic rate is, the bigger the user traffic weight becomes. So the high traffic rate user has greater probability to be fed back, which can support different traffic rate. Simulation results show that the proposed feedback mechanism can bring more feedback sub-carriers to the user with high traffic rate.
\end{abstract}

Index Terms: Feedback reduction; traffic rate; OFDM; relay

(C) 2011 Published by MECS Publisher. Selection and/or peer review under responsibility of the Research Association of Modern Education and Computer Science

\section{Introduction}

Relaying and OFDMA have gained a lot of interests in the wireless communication research community. By introducing relay, the system capacity can be improved and the coverage area can be extended. OFDMA is a multiple access technique, which can mitigate the problems of frequency of selectivity and intersymbol interference [1].

The uplink feedback load has gradually become an indicator of system performance evaluation. Therefore, how to reduce system feedback while ensuring user performance becomes a research difficulty. In [2], the threshold is set from the aspects of feedback outage probability, capacity loss, and feedback utility. Resource management and limited feedback theory in OFDM system is studied in [3].Based on feedback outage probability, threshold setting is dependent on user weight which is decided by user queue and delay. In [4], downlink packet transmission with different traffic rate is researched. In order to reduce uplink feedback load, the user whose channel gain is bigger than threshold will be fed back with a probability that is decided by user

* Corresponding author.

E-mail address: chenqb@cqupt.edu.cn, jianggjian@126.com, tangl@cqupt.edu.cn 
traffic rate, which supports different traffic rate. But in multiuser OFDM relay system, there is no feedback mechanism that based on outage probability to reduce feedback load, let alone a feedback mechanism that supports different user traffic rate.

Feedback mechanism based on outage probability in multiuser OFDM relay system is proposed. In order to support different traffic rate, user channel gain is weighted by traffic rate. The user whose traffic rate is high will have larger weighted gain. Therefore, after compared with threshold, the user will get more feedback subcarriers and obtain a higher transmission rate.

\section{System Model}

As shown in Fig. 1, the base station communicates with $M$ users with the assistance of a relay. In the BS, queues which send data in accordance with FIFO principle are established for each user. Assume that the data arrives to the BS with a fixed rate and the traffic rate of each user is different. A slot is divided into two sub-slots in the TDD system. In the first sub-slot BS sends data (BS-RS, BS-MS) and in the second sub-slot RS sends data (RS-MS). For two-hop user, the first hop is the link between BS to RS and the second hop is the link between RS-MS. Assume that there are $N$ sub-carriers in the system and the total system bandwidth is $B$. There are $M_{1}$ direct users in the set $U_{D}$ and $M_{2}$ relay users in the set $U_{R}$. BS does not collaborate with the relay.

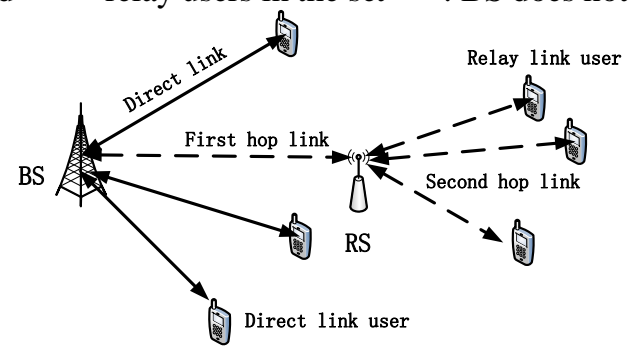

Figure 1. system structure

The capacity of direct user $k_{D}$ on the sub-carrier $n$ is

$$
r_{k_{D}, n}=\frac{B}{N} \log _{2}\left(1+p_{D} g_{k_{D}, n}^{2} /\left(\Gamma \sigma^{2}\right)\right)
$$

The capacity of relay user $k_{R}$ on the sub-carrier $n$ through the relay is

$$
r_{k_{R}, n}^{\prime}=\frac{B}{N} \log _{2}\left(1+p_{R} g_{k_{R}, n}^{2} /\left(\Gamma \sigma^{2}\right)\right)
$$

And the capacity of the relay on the sub-carrier $n$ is

$$
r_{n}=\frac{B}{N} \log _{2}\left(1+p_{D} g_{n}^{2} /\left(\Gamma \sigma^{2}\right)\right)
$$

The date rate of relay user on each sub-carrier is determined by the minimum value of the two hops, so

$$
r_{k_{R}, n}=\min \left\{r_{k_{R}, n}^{\prime}, r_{n}\right\}
$$


$\sigma^{2}$ is additive white Gaussian noise and $\Gamma$ equals to $-\ln \left(5^{*} B E R\right) / 1.5$. $p_{D}$ and $p_{R}$ are the power of BS and RS on each sub-carrier, respectively. $g_{k_{D}, n}$ is the gain of $k_{D}$ on sub-carrier $n, g_{k_{R}, n}$ is the gain of $k_{R}$ on $n$ and $g_{n}$ is the gain of the relay on sub-carrier $n . g_{k_{D}, n}$ and $g_{k_{R}, n}$ subject to the same Rayleigh distribution and are not changed during every slot. In general, relay is close to BS and the communication condition is well, so assume that $g_{n}$ is larger than $g_{k_{R}, n}$. $a_{k_{R}, n}$ and $a_{k_{D}, n}$ are index of sub-carrier allocation.

\section{Feeback Mechanism Supporting User Traffic Rate and Performance Analysis}

Assume that the channel gain of BS-MS link and RS-MS link subject to the same Rayleith distribution. As the channel condition of the BS-RS link is better than that of RS-MS link, the relay user rate on each sub-carrier depends on the channel gain of the second hop link in DF relay link. Therefore, if the sub-carrier is allocated to relay user, the data rate is the rate of the second hop link. Then the availability rate of each sub-carrier is the data rate of the user whose channel gain is the best among the direct feedback users and relay feedback users. The average rate of each sub-carrier is derived based on above analysis.

\section{A. Feedback Mechanism Supporting User Traffic Rate}

In order to support different traffic rate, define user traffic weight as

$$
a_{k}=\frac{\Psi_{k}}{\frac{1}{N} \sum_{k=1}^{K} \Psi_{k}}
$$

Before feedback, user gain is weighted, i.e. user gain is multiplied by the detected user traffic weight. Then the user weighted gain is compared with the threshold. If the former is bigger, the user will be fed back, otherwise, not be fed back. The user whose traffic rate is high will have larger traffic weight, so under the same condition it will obtain more feedback sub-carriers to support high traffic rate. Assume that user traffic rate is fixed, the user can handle the user traffic weight as a constant.

Feedback mechanism is described as follows:

Step1 BS calculates user traffic weight according to the user traffic rate and then informs it to RS.

Step2 RS broadcasts its threshold and user traffic weight to relay users.

Step3 Relay users multiply the detected channel gain with the traffic weight to obtain the weighed channel gain. Then compare the weighted gain with the threshold. If the former is bigger, the user will be fed back, otherwise, the user will be not.

Step4 BS broadcasts its threshold to direct users.

Step5 The direct users determine whether to feed back according to step3. At the same time, RS sends its feedback users to BS.

\section{B. $\quad$ Threshold Setting}

Threshold setting has a significant impact on system performance and feedback overhead. We discuss how to set the threshold based on feedback outage probability.

Take the relay user feedback as an example. Assume that all channel gains subject to Rayleigh distribution, and the probability distribution function is

$$
p(g)=\frac{g}{\delta^{2}} e^{-\frac{g^{2}}{2 \delta^{2}}}
$$


The probability that user channel gain is less than $x$ is

$$
P(g<x)=\int_{0}^{x} p(g) d g=1-e^{-\frac{x^{2}}{2 \delta^{2}}}
$$

Then the probability that all relay user gains are less than $x$ is

$$
\begin{aligned}
P_{M_{1}}(x) & =P_{M_{1}}\left(g_{1}, \cdots, g_{M_{1}}<x\right) \\
& =\prod_{k=1}^{M_{1}} P(g<x)=[P(g<x)]^{M_{1}} \\
& =\left(1-e^{-\frac{x^{2}}{2 \delta^{2}}}\right)^{M_{1}}
\end{aligned}
$$

Let $x$ be relay threshold, the above expression is the outage probability of relay feedback. So the outage probability is

$$
P_{\text {out } R}=P_{M_{1}}\left(g_{\text {thR }}\right)=\left(1-e^{-\frac{g_{\text {thR }}^{2}}{2 \delta^{2}}}\right)^{M_{1}}
$$

From (9), we can calculate the relay threshold as

$$
g_{\text {thR }}=\left[-2 \delta^{2} \ln \left(1-P_{\text {outR }} 1 / M_{1}\right)\right]^{1 / 2}
$$

Observe user traffic weight $a_{k}$, we know that $a_{k}$ fluctuates based on 1. When user traffic rate is same, $a_{k}$ equals to 1 . Based on this, the feedback mechanism which supports user traffic rate can use the threshold in (10) directly.

\section{Average Number of Feedback Users}

The weighted channel gain is

$$
h_{k}=a_{k} g_{k}
$$

And its probability density function is

$$
p(h)=\frac{h}{a^{2} \delta^{2}} e^{-\frac{h^{2}}{2 a^{2} \delta^{2}}}
$$

The probability that user weighted channel gain is greater than the threshold $g_{\text {thR }}$ is

$$
P=P\left(h>g_{t h R}\right)=\int_{g_{t h}}^{\infty} p(h) d h=e^{-\frac{g_{t h R}^{2}}{2 a^{2} \delta^{2}}}
$$

As user channel fades independently, the probability $P_{k}$ that $k$ users are fed back is

$$
\begin{aligned}
P_{k} & =\left(\begin{array}{l}
M_{1} \\
k
\end{array}\right) P^{k}(1-P)^{M_{1}-k} \\
& =\left(\begin{array}{l}
M_{1} \\
k
\end{array}\right) \prod_{i=1}^{k} e^{-\frac{g_{t h R}{ }^{2}}{2 a_{i}^{2} \delta^{2}}} \prod_{i=k+1}^{M_{1}}\left(1-e^{-\frac{g_{t h R}{ }^{2}}{2 a_{i}^{2} \delta^{2}}}\right)
\end{aligned}
$$

The average number of RS feedback users of each sub-carrier in the second sub-slot is 


$$
\begin{aligned}
\overline{M_{1}} & =\sum_{k=0}^{M_{1}} P_{k} \square k=\sum_{k=0}^{M_{1}}\left(\begin{array}{l}
M_{1} \\
k
\end{array}\right) P^{k}(1-P)^{M_{1}-k} \square k \\
& =\sum_{k=0}^{M_{1}}\left(\begin{array}{l}
M_{1} \\
k
\end{array}\right) \prod_{i=1}^{k} e^{-\frac{g_{t h R}^{2}}{2 a_{i}^{2} \delta^{2}}} \prod_{i=k+1}^{M_{1}}\left(1-e^{-\frac{g_{t h R}^{2}}{2 a_{i}^{2} \delta^{2}}}\right) \square k
\end{aligned}
$$

As the same as the second sub-slot, the average number of BS feedback users of each sub-carrier in the first sub-slot is

$$
\begin{aligned}
\overline{M_{2}} & =\sum_{k=0}^{M_{2}} P_{k} \square k=\sum_{k=0}^{M_{2}}\left(\begin{array}{l}
M_{2} \\
k
\end{array}\right) P^{k}(1-P)^{M_{2}-k} \square k \\
& =\sum_{k=0}^{M_{2}}\left(\begin{array}{l}
M_{2} \\
k
\end{array}\right) \prod_{i=1}^{k} e^{-\frac{g_{t h B^{2}}}{2 a_{i}^{2} \delta^{2}}} \prod_{i=k+1}^{M_{2}}\left(1-e^{-\frac{g_{t h B^{2}}}{2 a_{i}^{2} \delta^{2}}}\right) \square k
\end{aligned}
$$

Therefore, the total number of feedback users of each sub-carrier is

$$
\begin{aligned}
& M_{\text {tot }}=N M_{\text {sub }} \\
& =N\left(\sum_{k=0}^{M_{1}} k\left\lfloor\begin{array}{l}
M_{1} \\
k
\end{array}\right) \prod_{i=1}^{k} e^{-\frac{g_{h k}{ }^{2}}{2 a_{i}^{2} \delta^{2}}} \prod_{i=k+1}^{M_{1}}\left(1-e^{-\frac{g_{h h R^{2}}}{2 a_{i}^{2} \delta^{2}}}\right)\right. \\
& \left.+\sum_{k=0}^{M_{2}} k\left\{\begin{array}{l}
M_{2} \\
k
\end{array}\right) \prod_{i=1}^{k} e^{-\frac{g_{u b}{ }^{2}}{2 a_{i}^{2} \delta^{2}}} \prod_{i=k+1}^{M_{2}}\left(1-e^{-\frac{g_{h b}{ }^{2}}{2 a_{i}^{2} \delta^{2}}}\right)\right)
\end{aligned}
$$

\section{Average Available Data Rate}

Considering the average available data rate of each sub-carrier

$$
C=E\left[\log _{2}\left(1+\rho g^{2}\right)\right]
$$

According to [5], let $g=x$, then the probability density function that $g_{\max }=\max g(=x)$ is

$$
\begin{aligned}
p_{g_{\max }}(x) & =\sum_{k=1}^{M}\left(p_{k}(x) \prod_{j=1 ; j \neq k}^{M} F_{j}(x)\right) \\
& =M \frac{x}{\delta^{2}} e^{-\frac{x^{2}}{2 \delta^{2}}}\left(1-e^{-\frac{x^{2}}{2 \delta^{2}}}\right)^{M-1}
\end{aligned}
$$

Where $\rho=p /\left(\Gamma \sigma^{2}\right), p_{k}(x)$ is the probability density function of $g$ and $F_{j}(x)$ is the cumulative distribution function of $g$. From (18) and (19), we can obtain that the average available data rate of each subcarrier is

$$
\begin{aligned}
C & =\int_{g_{t h}}^{\infty} \log _{2}\left(1+\rho x^{2}\right) d p_{g_{\max }}(x) \\
& =\int_{g_{t h}}^{\infty} \log _{2}\left(1+\rho x^{2}\right) d M \frac{x}{\delta^{2}} e^{-\frac{x^{2}}{2 \delta^{2}}}\left(1-e^{-\frac{x^{2}}{2 \delta^{2}}}\right)^{M-1}
\end{aligned}
$$

Therefore, the available data rate of system is

$$
C_{\text {tot }}=N C
$$


When considering user traffic rate, the probability density function of user weighted gain is

$$
p\left(h_{k}\right)=\frac{h_{k}}{a_{k}^{2} \delta^{2}} e^{-\frac{h_{k}^{2}}{2 a_{k}^{2} \delta^{2}}}
$$

Because of different traffic weight $a_{k}$, the distribution of user weighted gain is different, the above derivation can not be used when supporting user traffic rate. ${ }{ }_{k}$ is a constant which fluctuates around 1 and the feedback is more dependent on user channel gain, so the average available data rate can be expressed approximately as (20). However, there may occur that the weighted channel gain of the fed back user is the biggest while the channel gain is not the biggest. In this situation, the average available data rate of each sub-carrier will be reduced.

\section{Simulation Results}

We consider a system with a single relay and multiple users. The number of the sub-carriers is 64 and the bandwidth of each sub-carrier is $0.1 \mathrm{MHz}$. The system power is allocated equally and the power of each subcarrier is $0.5 \mathrm{~W}$. The channel gain subjects to Rayleigh distribution with the variance of 2 . The user traffic rate is fixed.

Fig. 2 analyzes the average number of feedback users of each sub-carrier. When feedback outage probability is 0.1 and the total users is 100 , the average number of feedback users is 6 , which reduces the feedback load by 94\%. In addition, the increase of users does not make the feedback users increase simultaneously but tend to a fixed value. The reason is that the threshold which becomes bigger as the increase of users limits user feedback.
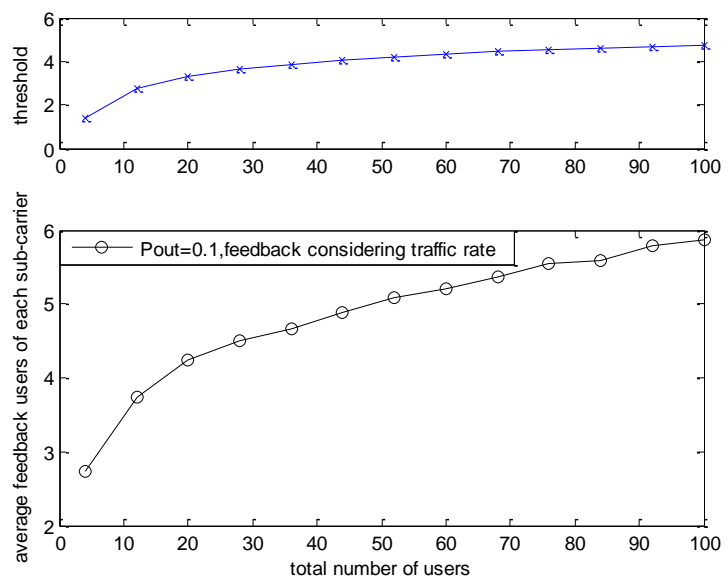

Figure 2. Number of users and number of feedback users

The user traffic rate is different and the user traffic weight reflects the user traffic rate. Their trends are same. Fig. 3 analyzes the relationship between user traffic rate and the number of feedback sub-carriers. Assume there are 8 users. It is user traffic weight in the following sub-figure; in the above sub-figure the blue line is the average number of feedback sub-carriers of each user without considering user traffic rate. It is almost a straight line. The red line represents the feedback mechanism considering user traffic rate. The traffic rate of the fourth user is the biggest and the number of sub-carriers which feed the fourth user back is the most whereas that of the second user is the least. This shows that the proposed feedback mechanism can support different user traffic rate. 


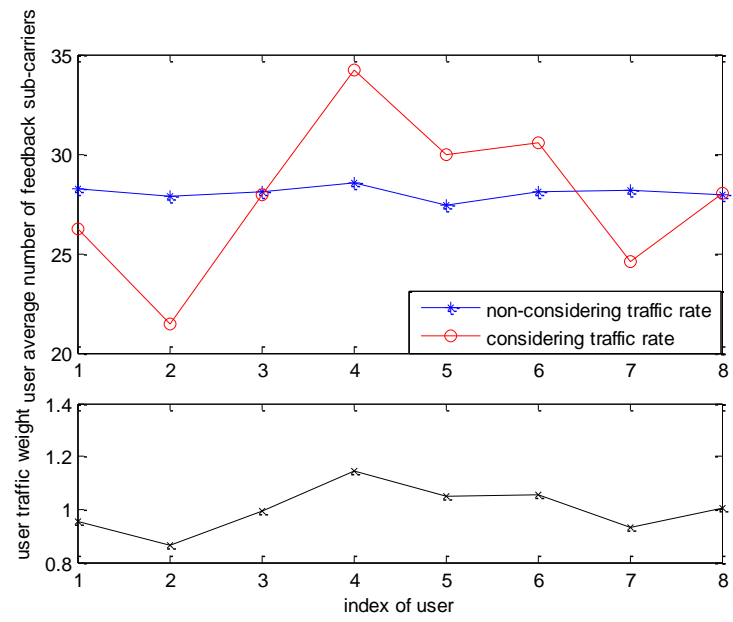

Figure 3. Traffic rate and the number of feedback sub-carriers

Fig. 4 shows the average data rate of each sub-carrier. Assume the outage probability is 0.1 . The simulation results show that the loss of the capacity of each sub-carrier is about $10 \%$, which is consistent with the outage probability. In addition, the loss considering traffic rate is larger, which is consistent with the analysis in the text. When considering traffic rate, there are many fluctuations which are affected by user traffic rate. The user with high traffic rate and low channel gain receives much more feedback, which causes the decline of the data rate.

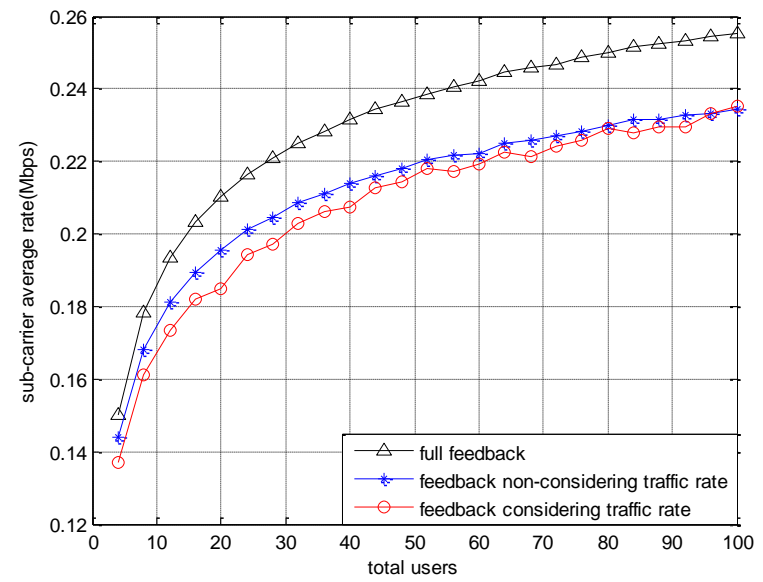

Figure 4. Number of users and average rate of sub-carriers

\section{Conclusion}

Uplink feedback has become a performance index in OFDM relay system. A feedback mechanism that supports user traffic rate is proposed in this paper. Based on feedback outage probability, user channel gain is weighted before user feedback, and then the weighted gain is compared with the threshold. In the same condition, the user with high traffic rate can obtain more feedback sub-carriers, which can support different traffic rate. Simulation results show that the proposed feedback mechanism can reduce feedback while maintaining the different user traffic rate. 


\section{Acknowledgment}

This work was supported by the National Key Technology R\&D Program (2008BAH30B10), the National Science and Technology Major Special Project of China (2008ZX03003-005), the Natural Science Foundation of China (60972070), the Natural Science Foundation of Chongqing, China (CSTC2009BA2090) and the Foundation of Chongqing Educational Committee, China (KJ100514).

\section{References}

[1] Bin Fan, Wenbo Wang, Yicheng Lin, Lin Huang and Kan Zheng, "Subcarrier allocation for OFDMA relay networks with proportional fair constraint”, Communication, 2009. ICC'09. Dresden:2009.6:1-5.

[2] Tang Z H, Zhu Y T and Wei G, "Threshold setting of channel limited feedback in OFDM", Journal of Electronics \& Information Technology, 2007,29(4): 933-937(in Chinese)

[3] YANG Ruizhe, YUAN Chaowei, TENG Yinglei and ZHAGN Yanhua, "Feedback Threshold with Guaranteed QoS in Multiuser OFDM Systems", THE JOURNAL OF CHINA UNIVERSITIES OF POSTS AND TELECOMMUNICATIONS, 2009,16 (2) : 24-28.

[4] Seung Young Park, "Performance of Differentiated Rate Scheduling Using Contention-Based CSI Feedback", VEHICULAR TECHNOLOGY, 2010,59(2):3143-3148.

[5] Yi Shi, Wei Zhang and Ben Letaief,K, "Cooperative Multiplexing and Scheduling in Wireless Relay Networks”, Communication, 2008. ICC’08. Beijing:2008,5:3034-3038. 